\title{
Short-distance constraints on hadronic light-by-light scattering in the anomalous magnetic moment of the muon
}

\author{
Gilberto Colangelo® ${ }^{1, *}$ Franziska Hagelstein $\odot,{ }^{1, \dagger}$ Martin Hoferichter ${ }^{2,1, \$}$ \\ Laetitia Laub $\oplus^{1, \S}$ and Peter Stoffer $\oplus^{3, \|}$ \\ ${ }^{1}$ Albert Einstein Center for Fundamental Physics, Institute for Theoretical Physics, \\ University of Bern, Sidlerstrasse 5, CH-3012 Bern, Switzerland \\ ${ }^{2}$ Institute for Nuclear Theory, University of Washington, Seattle, Washington 98195-1550, USA \\ ${ }^{3}$ Department of Physics, University of California at San Diego, La Jolla, California 92093, USA
}

(Received 1 November 2019; accepted 10 February 2020; published 5 March 2020)

\begin{abstract}
A key ingredient in the evaluation of hadronic light-by-light (HLBL) scattering in the anomalous magnetic moment of the muon $(g-2)_{\mu}$ concerns short-distance constraints that follow from QCD by means of the operator product expansion. Here we concentrate on the most important such constraint, in the longitudinal amplitudes, and show that it can be implemented efficiently in terms of a Regge sum over excited-pseudoscalar states, constrained by phenomenological input on masses, two-photon couplings, as well as short-distance constraints on HLBL scattering and the pseudoscalar transition form factors. Our estimate of the effect of the longitudinal short-distance constraints on the HLBL contribution is $\Delta a_{\mu}^{\mathrm{LSDC}}=13(6) \times 10^{-11}$. This is significantly smaller than previous estimates, which mostly relied on an ad-hoc modification of the pseudoscalar poles and led to up to a $40 \%$ increase with respect to the nominal pseudoscalar-pole contributions, when evaluated with modern input for the relevant transition form factors. We also comment on the status of the transversal short-distance constraints and, by matching to perturbative QCD, argue that the corresponding correction will be significantly smaller than its longitudinal counterpart.
\end{abstract}

DOI: 10.1103/PhysRevD.101.051501

\section{INTRODUCTION}

The precision of the Standard-Model (SM) prediction for the anomalous magnetic moment of the muon, $a_{\mu}=$ $(g-2)_{\mu} / 2$, is limited by hadronic contributions. Already at the level of the current experiment [1]

$$
a_{\mu}^{\exp }=116592089(63) \times 10^{-11},
$$

estimates of the hadronic effects are crucial in evaluating the significance of the tension with the SM value, at the level of $3.5 \sigma$. With the forthcoming Fermilab E989 experiment [2], promising an improvement by a factor of 4 , as well as the E34 experiment at J-PARC [3], the SM model evaluation needs to follow suit.

\footnotetext{
*gilberto@itp.unibe.ch

hagelstein@itp.unibe.ch

*mhofer@uw.edu

§laub@itp.unibe.ch

"pstoffer@ucsd.edu
}

Published by the American Physical Society under the terms of the Creative Commons Attribution 4.0 International license. Further distribution of this work must maintain attribution to the author(s) and the published article's title, journal citation, and DOI. Funded by SCOAP.
To this end, the relevant matrix elements need to be calculated either directly from QCD or be constrained by experimental data. The latter approach has traditionally been followed for hadronic vacuum polarization (HVP), which requires the two-point function of two electromagnetic currents and can be reconstructed from the cross section of $e^{+} e^{-} \rightarrow$ hadrons [4-8]. More recently, evaluations in lattice QCD have made significant progress [9-15], but are not yet at the level of the data-driven, dispersive approach.

Next to HVP, the second-largest contribution to the uncertainty arises from hadronic light-by-light (HLBL) scattering. While also in this case progress in lattice QCD is promising [16-18], another key development in recent years concerns the phenomenological evaluation, i.e., the use of dispersion relations to remove the reliance on hadronic models, either directly for the required four-point function that defines the HLBL tensor [19-24], the Pauli form factor [25], or in terms of sum rules [26-30]. In particular, organizing the calculation in terms of dispersion relations for the HLBL tensor has led to a solid understanding of the contributions related to the lowest-lying singularitiesthe single-particle poles from $P=\pi^{0}, \eta, \eta^{\prime}$ and cuts from two-pion intermediate states-largely because the hadronic quantities determining the strength of these 
singularities, the $P \rightarrow \gamma^{*} \gamma^{*}$ transition form factors [31-36] and the helicity amplitudes for $\gamma^{*} \gamma^{*} \rightarrow \pi \pi$ [37-42], respectively, can be provided as external input quantities, in a similar spirit as the $e^{+} e^{-} \rightarrow$ hadrons cross section for HVP. Higher-order iterations of HVP $[4,43,44]$ and HLBL [45] are already sufficiently under control.

For both HVP and HLBL, data-driven evaluations of the hadronic corrections to $(g-2)_{\mu}$ are fundamentally limited by the fact that experimental input is only available in a given energy range, so that the tails of the dispersion integrals have to be estimated by other means, most notably short-distance constraints as derived from perturbative QCD (pQCD). In addition, even for HVP, shortdistance constraints have been used for energies as low as $2 \mathrm{GeV}$ as a supplement to (and check of) experiment, with good agreement found between the pQCD prediction and data in between resonances $[4,8]$. For HLBL scattering such constraints become even more important given the limited information on the HLBL tensor for intermediate and high energies.

Two kinematic configurations are relevant for the HLBL contribution, one in which all photon virtualities $Q_{i}^{2}$ are large, and a second in which one of the nonvanishing virtualities remains small compared to the others $Q_{3}^{2} \ll Q_{1}^{2} \sim Q_{2}^{2}$. Recently, it was shown that the former situation can be addressed in a systematic operator product expansion (OPE), in which the pQCD quark loop emerges as the first term in the expansion [46]. The second configuration is related to so-called mixed regions in the $g-2$ integral, i.e., integration regions in which asymptotic arguments only apply to a subset of the kinematic variables, while hadronic physics may still be relevant for others. A key insight derived in [47] was that such effects can also be constrained with an OPE, by reducing the HLBL tensor to a vector-vector-axialvector $(V V A)$ three-point function and using known results for the corresponding anomaly and its (non-) renormalization [48-53]. The explicit implementation suggested in [47] relied on the observation that both the OPE constraint and the normalization are satisfied if the momentum dependence of the singly virtual form factor describing the pseudoscalar-pole contribution is neglected. However, such a modification is not compatible with a description based on dispersion relations for the HLBL tensor.

Here, we suggest to implement the corresponding longitudinal short-distance constraints in terms of excited-pseudoscalar states. As we will show, not only can the asymptotic limits be implemented in a fairly economical manner, but the critical mixed regions can be constrained by phenomenological input for the masses and two-photon couplings of the lowest pseudoscalar excitations. The model dependence can be further reduced by matching to the $\mathrm{pQCD}$ quark loop, which, in addition, allows one to gain some insights into the scale where hadronic and pQCD-based descriptions should meet.

\section{OPE CONSTRAINTS ON HLBL SCATTERING}

The HLBL tensor is defined as the four-point function

$$
\begin{aligned}
\Pi^{\mu \nu \lambda \sigma}\left(q_{1}, q_{2}, q_{3}\right)= & -i \int d^{4} x d^{4} y d^{4} z e^{-i\left(q_{1} \cdot x+q_{2} \cdot y+q_{3} \cdot z\right)} \\
& \times\left\langle 0\left|T\left\{j_{\mathrm{em}}^{\mu}(x) j_{\mathrm{em}}^{\nu}(y) j_{\mathrm{em}}^{\lambda}(z) j_{\mathrm{em}}^{\sigma}(0)\right\}\right| 0\right\rangle
\end{aligned}
$$

of four electromagnetic currents

$$
j_{\mathrm{em}}^{\mu}=\bar{q} \mathcal{Q} \gamma^{\mu} q, \quad \mathcal{Q}=\operatorname{diag}\left(\frac{2}{3},-\frac{1}{3},-\frac{1}{3}\right),
$$

where $q_{i}$ denote the photon virtualities, $q_{4}=q_{1}+q_{2}+q_{3}$, and $q=(u, d, s)^{T}$ the quark fields. We work with the decomposition into scalar functions $\Pi_{i}$,

$$
\Pi^{\mu \nu \lambda \sigma}=\sum_{i=1}^{54} T_{i}^{\mu \nu \lambda \sigma} \Pi_{i},
$$

derived in [22,24] following the general principle established by Bardeen, Tung [54], and Tarrach [55] (BTT). The contribution to $(g-2)_{\mu}$ then follows via

$$
\begin{aligned}
a_{\mu}^{\mathrm{HLBL}}= & \frac{2 \alpha^{3}}{3 \pi^{2}} \int_{0}^{\infty} d Q_{1} \int_{0}^{\infty} d Q_{2} \int_{-1}^{1} d \tau \sqrt{1-\tau^{2}} Q_{1}^{3} Q_{2}^{3} \\
& \times \sum_{i=1}^{12} T_{i}\left(Q_{1}, Q_{2}, \tau\right) \bar{\Pi}_{i}\left(Q_{1}, Q_{2}, Q_{3}\right),
\end{aligned}
$$

where $Q_{i}^{2}=-q_{i}^{2}$ are the Wick-rotated virtualities, $Q_{3}^{2}=Q_{1}^{2}+Q_{2}^{2}+2 Q_{1} Q_{2} \tau$, the $\bar{\Pi}_{i}$ refer to certain linear combinations of $\Pi_{i}$, and the $T_{i}$ are known kernel functions [22,24].

In the limit where all $Q_{i}^{2}$ are large, the calculation from [46] proves the earlier statement of [47] that the pQCD quark loop arises as the first term in a systematic OPE. In particular, this implies the constraint

$$
\lim _{Q \rightarrow \infty} Q^{4} \bar{\Pi}_{1}(Q, Q, Q)=-\frac{4}{9 \pi^{2}} .
$$

The second kinematic configuration [47], $Q^{2} \equiv Q_{1}^{2} \sim$ $Q_{2}^{2} \gg Q_{3}^{2}$, when expressed in BTT basis, leads to the constraint

$$
\lim _{Q_{3} \rightarrow \infty} \lim _{Q \rightarrow \infty} Q^{2} Q_{3}^{2} \bar{\Pi}_{1}\left(Q, Q, Q_{3}\right)=-\frac{2}{3 \pi^{2}} .
$$


The latter result can be derived by considering the $V V A$ triangle anomaly and its nonrenormalization theorems [48-53]. Its constraint on $\bar{\Pi}_{1}$ (and, by crossing symmetry, $\bar{\Pi}_{2}$ ) corresponds to the longitudinal amplitudes in the $V V A$ matrix element and we will therefore refer to $\bar{\Pi}_{1,2}$ as the longitudinal amplitudes and, accordingly, their constraints as longitudinal short-distance constraints. Further, the limit (7) is intimately related to the pseudoscalar poles

$$
\bar{\Pi}_{1}^{P \text { pole }}=\frac{F_{P \gamma^{*} \gamma^{*}}\left(q_{1}^{2}, q_{2}^{2}\right) F_{P \gamma \gamma^{*}}\left(q_{3}^{2}\right)}{q_{3}^{2}-M_{P}^{2}},
$$

where $P=\pi^{0}, \eta, \eta^{\prime}$, and the doubly virtual $F_{P \gamma^{*} \gamma^{*}}\left(q_{1}^{2}, q_{2}^{2}\right)$ and singly virtual $F_{P \gamma \gamma^{*}}\left(q_{3}^{2}\right)$ transition form factors determine the residue of the poles. They are subject to short-distance constraints themselves, for the pion we have the asymptotic constraint [56]

$$
\lim _{Q^{2} \rightarrow \infty} Q^{2} F_{\pi^{0} \gamma^{*} \gamma^{*}}\left(-Q^{2},-Q^{2}\right)=\frac{2 F_{\pi}}{3},
$$

as well as the Brodsky-Lepage limit [57-59]

$$
\lim _{Q^{2} \rightarrow \infty} Q^{2} F_{\pi^{0} \gamma \gamma^{*}}\left(-Q^{2}\right)=2 F_{\pi}
$$

Together with the normalization

$$
F_{\pi^{0} \gamma \gamma}=\frac{1}{4 \pi^{2} F_{\pi}}
$$

the former shows that if $F_{\pi^{0} \gamma \gamma^{*}}\left(q_{3}^{2}\right) \rightarrow F_{\pi^{0} \gamma \gamma}$ in (8), the pion decay constant $F_{\pi}$ would drop out and the pion would account for $-1 /\left(6 \pi^{2}\right)$ in (7). Similarly, $\eta$ and $\eta^{\prime}$ would provide the remaining $-1 /\left(2 \pi^{2}\right)$. This is the essence of the model suggested in $[47,60]$.

However, a constant singly virtual transition form factor cannot be justified within a dispersive approach for general HLBL scattering. Instead, one would need to consider dispersion relations in the photon virtualities $q_{i}^{2}$ already in reduced $g-2$ kinematics, and even then the residue would involve $F_{\pi^{0} \gamma \gamma^{*}}\left(M_{P}^{2}\right)$, not the normalization itself. Further, when writing dispersion relations in the $q_{i}^{2}$ for $g-2$ kinematics, there is no clear separation between the singularities of the HLBL amplitude and those generated by hadronic intermediate states directly coupling to individual electromagnetic currents, such as $2 \pi$ states. In the dispersive approach for general HLBL scattering the latter appear only in the transition form factors, which factor out and can be treated as external input quantities. In this sense, neglecting the momentum dependence of the singly virtual transition form factor without at the same time accounting for the additional cuts, leads to a distortion of the low-energy properties of the HLBL tensor.

Instead, we propose here a solution based on a remark already made in [47]: while a finite number of pseudoscalar poles, due to (11), cannot fulfill the OPE constraint (7), an infinite series potentially can. The basic idea can be illustrated for large- $N_{c}$ Regge models of the transition form factor itself $[61,62]$, which assume a radial Regge trajectory to describe the masses of excited vector mesons,

$$
M_{V(n)}^{2}=M_{V}^{2}+n \sigma_{V}^{2},
$$

and rely on the ansatz:

$$
\begin{aligned}
F_{P \gamma^{*} \gamma^{*}}\left(-Q^{2},-Q^{2}\right) & \propto \sum_{n=0}^{\infty} \frac{1}{\left[Q^{2}+M_{V(n)}^{2}\right]^{2}} \\
& =\frac{1}{\sigma_{V}^{4}} \psi^{(1)}\left(\frac{M_{V}^{2}+Q^{2}}{\sigma_{V}^{2}}\right) \sim \frac{1}{Q^{2}},
\end{aligned}
$$

with $\psi^{(1)}$ the trigamma function and the Regge slope $\sigma_{V}$. In this way, the infinite sum produces the correct asymptotic behavior (10), even though none of the individual terms do.

One may wonder about the fate of the infinite sum over excited-pseudoscalar states in the chiral limit, given that their decay constants are expected to vanish with the quark masses. We show below how the matching to pQCD removes the model dependence regarding which states are used to satisfy the short-distance constraints, so that the implementation in terms of pseudoscalar excitations mainly adds an estimate for the mixed-region contribution, driven by the phenomenology of the lowest excitations as well as the respective short-distance constraints.

\section{LARGE- $N_{c}$ REGGE MODEL}

In the following, we present a large- $N_{c}$-inspired Regge model in the pseudoscalar and vector-meson sectors of QCD that allows us to satisfy the short-distance constraints via an infinite sum of pseudoscalar-pole diagrams (see, e.g., [63-65] for the use of large- $N_{c}$ arguments to simultaneously fulfill low- and high-energy constraints). For brevity, we focus our description on the pion, referring for a complete and more detailed account to [66]. We start from a standard large- $N_{c}$ ansatz for the pion transition form factors as in (13), but differentiate between $\rho$ and $\omega$ trajectories, which are assumed to enter with diagonal couplings due to the wave function overlap $[61,62]$. In a first step, we seek an extension of this model that satisfies the constraints (9)-(11) for the transition form factor as well as (6) and (7) for the HLBL tensor 


$$
\begin{aligned}
F_{\pi(n) \gamma^{*} \gamma^{*}}\left(-Q_{1}^{2},-Q_{2}^{2}\right) & =\frac{1}{8 \pi^{2} F_{\pi}}\left\{( \frac { M _ { \rho } ^ { 2 } M _ { \omega } ^ { 2 } } { D _ { \rho ( n ) } ^ { 1 } D _ { \omega ( n ) } ^ { 2 } } + \frac { M _ { \rho } ^ { 2 } M _ { \omega } ^ { 2 } } { D _ { \rho ( n ) } ^ { 2 } D _ { \omega ( n ) } ^ { 1 } } ) \left[c_{\mathrm{anom}}\right.\right. \\
& \left.+\frac{1}{\Lambda^{2}}\left(c_{A} M_{+, n}^{2}+c_{B} M_{-, n}^{2}\right)+c_{\mathrm{diag}} \frac{Q_{1}^{2} Q_{2}^{2}}{\Lambda^{2}\left(Q_{+}^{2}+M_{\mathrm{diag}}^{2}\right)}\right] \\
& +\frac{Q_{-}^{2}}{Q_{+}^{2}}\left[c_{\mathrm{BL}}+\frac{1}{\Lambda^{2}}\left(c_{A} M_{-, n}^{2}+c_{B} M_{+, n}^{2}\right)\right] \\
& \left.\times\left(\frac{M_{\rho}^{2} M_{\omega}^{2}}{D_{\rho(n)}^{1} D_{\omega(n)}^{2}}-\frac{M_{\rho}^{2} M_{\omega}^{2}}{D_{\rho(n)}^{2} D_{\omega(n)}^{1}}\right)\right\},
\end{aligned}
$$

where $Q_{ \pm}^{2}=Q_{1}^{2} \pm Q_{2}^{2}, M_{ \pm, n}^{2}=\frac{1}{2}\left[M_{\omega(n)}^{2} \pm M_{\rho(n)}^{2}\right], D_{X}^{i}=$ $Q_{i}^{2}+M_{X}^{2}$, and $\Lambda=\mathcal{O}(1 \mathrm{GeV})$ a typical QCD scale. The five dimensionless parameters $c_{\text {anom }}, c_{A}, c_{B}, c_{\text {diag }}, c_{\mathrm{BL}}$ are used to fulfill all the constraints, while the remaining parameter $M_{\text {diag }}$ is adjusted to reproduce the ground-state $\pi^{0}$ transition form factor [33,34]. In the minimal model (14), we only allow $\pi(n)$ to couple to $\rho(n)$ and $\omega(n)$, i.e., the couplings are fully diagonal in the excitation numbers, while the effect of the eliminated vector-meson excitations is subsumed into a $Q_{i}^{2}$ dependence of the numerator multiplying the resonance propagators. In addition, we also considered an untruncated large- $N_{c}$ model, in which both the Regge summation in the transition form factor itself (13) and the HLBL tensor are retained, to assess the systematics in the large- $N_{c}$ ansatz [66]. Using the Regge slopes from [67] and the other input parameters from [68], we find that we can indeed reproduce well the $\pi^{0}$ transition form factor, which also ensures that effective-field-theory constraints on the pion-pole contribution to $(g-2)_{\mu}[69,70]$ are fulfilled [34]. Finally, the model predicts a two-photon coupling of the first excited pion, $\pi(1300)$, in line with its phenomenological bound $F_{\pi(1300) \gamma \gamma}<0.0544(71) \mathrm{GeV}^{-1}$ $[71,72]$.

Constructing a large- $N_{c}$ Regge model for $\eta^{(\prime)}$ proceeds along the same lines, but involves several complications. First, the $\rho$ and $\omega$ trajectories do not suffice to incorporate all constraints since due to the $I=0$ nature of $\eta^{(\prime)}$ only equal-mass combinations of vector mesons $(2 \rho, 2 \omega, 2 \phi)$ contribute to (14), so that only three model parameters survive. To provide sufficient freedom in satisfying all constraints the consideration of $\omega-\phi$ mixing cannot be avoided. In addition, $\eta-\eta^{\prime}$ mixing needs to be taken into account, both for the flavor decomposition of the shortdistance constraints as well as the relative weights of the vector-meson combinations in the transition form factors. The former is directly constrained by data on the transition form factors [32,73], but for the calculation of the weights, which we extract from effective pseudoscalar-vector-vector and photon-vector Lagrangians [74,75], it is more convenient to work with the phenomenological two-angle mixing scheme from [76,77]. We therefore use the latter everywhere. All variants are covered by the error analysis.

The resulting $\eta$ and $\eta^{\prime}$ transition form factors are in good agreement with experimental data in the singly virtual [78-81] and doubly virtual regions [82], as well as the fit results using Canterbury approximants [32]. Furthermore, there are some phenomenological constraints on the twophoton couplings for $\eta(1295)$ [83,84], $\eta(1405)$ [83-85], $\eta(1475)$ [85,86], $\eta(1760)$ [87], and $X(1835)$ [85,87], where $\eta(1475)$ and $\eta(1760)$ are actually seen in $\gamma \gamma$ collisions, while for the others only limits are available. The detailed comparison depends on the assignment of these states into $\eta$ and $\eta^{\prime}$ trajectories, but the predictions of our model are compatible with either the assignment from [67,68] (our main choice) or the one from [88], see [66].

By construction, the ground-state pseudoscalarpole contributions to $(g-2)_{\mu}$ reproduce literature values [32-34,89] within errors, while the sum over excitedpseudoscalar poles leads to the increase:

$$
\begin{aligned}
\Delta a_{\mu}^{\pi \text {-poles }} & =2.7(0.4)_{\text {Model }}(1.2)_{\text {syst }} \times 10^{-11}, \\
\Delta a_{\mu}^{\eta \text {-poles }} & =\left.3.4_{-0.7}^{+0.9}\right|_{\text {Model }}(0.9)_{\text {syst }} \times 10^{-11}, \\
\Delta a_{\mu}^{\eta^{\prime} \text {-poles }} & =6.5(1.1)_{\text {Model }}(1.7)_{\text {syst }} \times 10^{-11},
\end{aligned}
$$

where the first error refers to the uncertainties propagated from the input parameters and the systematic error is estimated by comparison to an alternative untruncated large- $N_{c}$ Regge model [66]. Combining all pseudoscalars, we find

$$
\begin{aligned}
\Delta a_{\mu}^{\text {PS-poles }} & =\Delta a_{\mu}^{\pi \text {-poles }}+\Delta a_{\mu}^{\eta \text {-poles }}+\Delta a_{\mu}^{\eta^{\prime} \text {-poles }} \\
& =\left.12.6_{-1.5}^{+1.6}\right|_{\text {Model }}(3.8)_{\text {syst }} \times 10^{-11} \\
& =12.6(4.1) \times 10^{-11}
\end{aligned}
$$

This result should be contrasted with the one suggested in [47] to satisfy the mixed-region short-distance constraint (using transition form factor models from [90]): $\left.\Delta a_{\mu}^{\text {PS-poles }}\right|_{\mathrm{MV}}=23.5 \times 10^{-11}$, which would become $38 \times$ $10^{-11}$ once updated with modern input for the transition form factors, and thus suggest an increase nearly 3 times as large as (16) or almost $40 \%$ of the nominal pseudoscalarpole contribution. Given that arguments following [47] have been included in previous compilations of HLBL [91], a central result of this work is that such a large increase does not occur if the short-distance constraints are implemented without compromising the low-energy properties of HLBL scattering.

\section{MATCHING TO PERTURBATIVE QCD}

Since, by construction, the sum over the pseudoscalar excitations fulfills the short-distance constraints, it has to match onto the pQCD quark loop for sufficiently large momentum transfers. In the upper plot of Fig. 1, the 

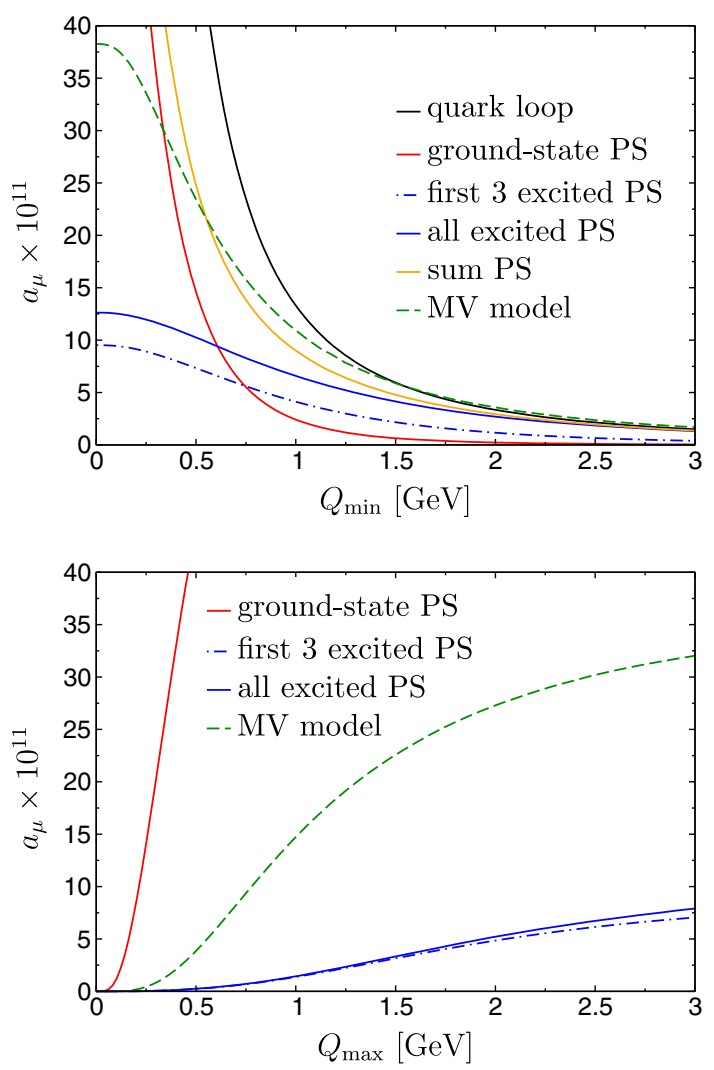

FIG. 1. The longitudinal part of the massless pQCD quark loop (black), the ground-state pseudoscalars (red), the sum of all excitations from the large- $N_{c}$ Regge model (blue), the first three excitations (blue dot-dashed), the sum of ground and excited states (orange), and the increase found in the Melnikov-Vainshtein model (green dashed). The upper plot shows the contribution to $a_{\mu}$ for $Q_{i} \geq Q_{\min }$, the lower for $Q_{i} \leq Q_{\max }$.

contribution to $(g-2)_{\mu}$ from the massless pQCD quark loop (black) and the pseudoscalar-pole contributions (sum of ground-state and excited states in orange) are compared after imposing a cutoff $Q_{\min } \leq Q_{i}$ in the integration: the matching occurs somewhere around $1.5-2 \mathrm{GeV}$. The lower plot, where the opposite cutoff $Q_{\max } \geq Q_{i}$ is imposed, shows that the contribution of the excited pseudoscalars (blue) to the low-energy region is very small and entirely saturated by the first few excitations (blue dot-dashed). These observations suggest to evaluate the asymptotic part of the integral $Q_{i} \geq Q_{\min }$ with $\mathrm{pQCD}$, to make explicit that this part of the result does not depend on the nature of hadronic states used in the implementation. Defining an optimal matching scale would require information on the uncertainty of the pQCD result. Here, we simply use a rough $20 \%$ estimate, which is the size of pQCD corrections for inclusive $\tau$ decays, a process that has a similar energy scale and has been studied in detail [92-97].

Together with the uncertainties from the Regge model, these considerations lead to a scale $Q_{\text {match }}=1.7 \mathrm{GeV}$. Varying this scale within $\pm 0.5 \mathrm{GeV}$ and adding the

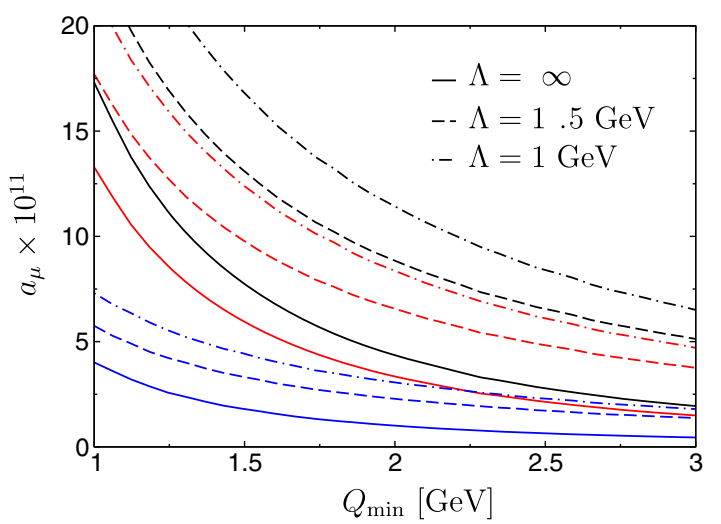

FIG. 2. Contribution of the massless pQCD quark loop to $a_{\mu}$ from the region $Q_{1,2} \geq Q_{\min }$, with the contribution from $Q_{3}$ below $Q_{\text {min }}$ damped by $Q_{3}^{2} /\left(Q_{3}^{2}+\Lambda^{2}\right)$ (plus crossed). The total contribution (black) is split into longitudinal (red) and transversal (blue) components. The limit $Q_{i} \geq Q_{\min }$ for all $Q_{i}$ is reproduced for $\Lambda \rightarrow \infty$.

systematic uncertainty from the comparison to the untruncated Regge model, we obtain as our final result:

$$
\begin{aligned}
\Delta a_{\mu}^{\mathrm{LSDC}} & =\left[8.7(5.5)_{\mathrm{PS}-\text { poles }}+4.6(9)_{\mathrm{pQCD}}\right] \times 10^{-11} \\
& =13(6) \times 10^{-11}
\end{aligned}
$$

for the increase of $(g-2)_{\mu}$ due to longitudinal shortdistance constraints. In particular, the lowest three pseudoscalar excitations, whose contribution is at least partly constrained by phenomenological input on masses and two-photon couplings, give $7.8 \times 10^{-11}$. Given that the most uncertain contribution, from $n>3$, thus amounts to only $10 \%$ of the total, the uncertainty estimate in (17) should be conservative enough to cover the remaining model dependence. In particular, the error in (17) includes an inflation of the Regge slope uncertainties by a factor three, to allow for systematic effects that might occur if other hadronic states were used to implement the shortdistance constraints. More recently, this expectation has been confirmed by models in holographic QCD based on a summation of an infinite tower of axial-vector resonances instead $[98,99]$, which despite very different assumptions and systematics yield results remarkably close to (17).

With the impact of the longitudinal short-distance constraints estimated as in (17), it is natural to inquire about the role of the transversal short-distance constraints. A first estimate could again be obtained by matching to pQCD. Figure 2 extends the integration region beyond $Q_{i} \geq Q_{\min }$ into the mixed region, but suppressing this additional contribution by a factor $Q_{3}^{2} /\left(Q_{3}^{2}+\Lambda^{2}\right)$, because otherwise part of the ground-state pseudoscalar contribution would be double counted. The longitudinal result is reproduced for scales around $\Lambda \sim Q_{\min } \sim 1.4 \mathrm{GeV}$, for which one would read off $\Delta a_{\mu}^{\mathrm{TSDC}} \sim 4 \times 10^{-11}$. Accordingly, we would 
expect the impact of the transversal short-distance constraints to be significantly less than that of the longitudinal ones.

We stress that the calculation presented here is complementary to higher-order calculations in $\mathrm{pQCD}$ and/or the OPE [46], which would allow one to improve the matching between hadronic implementations and a perturbative description. Similarly, more experimental guidance on the two-photon couplings of hadronic states in the 1-2 GeV region would be beneficial for the phenomenological analysis, not only for the excited pseudoscalars, but for axial-vector resonances as well, which outlines avenues for future work. We conclude that with the present analysis the biggest systematic uncertainty due to short-distance constraints has been reduced significantly, with the result that the asymptotic part of the HLBL tensor is under sufficient control for the first release from the Fermilab experiment.

\section{ACKNOWLEDGMENTS}

We thank R. Arnaldi, P. Bickert, J. Bijnens, S. Eidelman, A. Gérardin, C. Hanhart, S. Holz, B. Kubis, S. Leupold, J. Lüdtke, A. Manohar, V. Metag, M. Procura, E. Ruiz Arriola, P. Sanchez-Puertas, A. Uras, G. Usai, A. Vainshtein, and E. Weil for useful communication on various aspects of this work. Financial support by the DOE (Grants No. DE-FG02-00ER41132 and No. DE-SC0009919) and the Swiss National Science Foundation is gratefully acknowledged. M. H. is supported by an SNSF Eccellenza Professorial Fellowship (Project No. PCEFP2_181117).
[1] G. W. Bennett et al. (Muon $g-2$ Collaboration), Phys. Rev. D 73, 072003 (2006).

[2] J. Grange et al. (Muon $g-2$ Collaboration), arXiv: 1501.06858 .

[3] M. Abe et al., Prog. Theor. Exp. Phys. 2019, $053 \mathrm{C} 02$ (2019).

[4] A. Keshavarzi, D. Nomura, and T. Teubner, Phys. Rev. D 97, 114025 (2018).

[5] F. Jegerlehner, EPJ Web Conf. 199, 01010 (2019).

[6] G. Colangelo, M. Hoferichter, and P. Stoffer, J. High Energy Phys. 02 (2019) 006.

[7] M. Hoferichter, B.-L. Hoid, and B. Kubis, J. High Energy Phys. 08 (2019) 137.

[8] M. Davier, A. Hoecker, B. Malaescu, and Z. Zhang, arXiv:1908.00921.

[9] S. Borsanyi et al. (Budapest-Marseille-Wuppertal Collaboration), Phys. Rev. Lett. 121, 022002 (2018).

[10] T. Blum, P. A. Boyle, V. Gülpers, T. Izubuchi, L. Jin, C. Jung, A. Jüttner, C. Lehner, A. Portelli, and J. T. Tsang (RBC and UKQCD Collaborations), Phys. Rev. Lett. 121, 022003 (2018).

[11] D. Giusti, F. Sanfilippo, and S. Simula, Phys. Rev. D 98, 114504 (2018).

[12] E. Shintani and Y. Kuramashi (PACS Collaboration), Phys. Rev. D 100, 034517 (2019).

[13] C. T. H. Davies et al. (Fermilab Lattice, LATTICE-HPQCD, and MILC Collaborations), Phys. Rev. D 101, 034512 (2020).

[14] A. Gérardin, M. Cè, G. von Hippel, B. Hörz, H. B. Meyer, D. Mohler, K. Ottnad, J. Wilhelm, and H. Wittig, Phys. Rev. D 100, 014510 (2019).

[15] C. Aubin, T. Blum, C. Tu, M. Golterman, C. Jung, and S. Peris, Phys. Rev. D 101, 014503 (2020).

[16] T. Blum, N. Christ, M. Hayakawa, T. Izubuchi, L. Jin, C. Jung, and C. Lehner, Phys. Rev. Lett. 118, 022005 (2017).
[17] T. Blum, N. Christ, M. Hayakawa, T. Izubuchi, L. Jin, C. Jung, and C. Lehner, Phys. Rev. D 96, 034515 (2017).

[18] N. Asmussen, A. Gérardin, A. Nyffeler, and H. B. Meyer, SciPost Phys. Proc. 1, 031 (2019).

[19] M. Hoferichter, G. Colangelo, M. Procura, and P. Stoffer, Int. J. Mod. Phys. Conf. Ser. 35, 1460400 (2014).

[20] G. Colangelo, M. Hoferichter, M. Procura, and P. Stoffer, J. High Energy Phys. 09 (2014) 091.

[21] G. Colangelo, M. Hoferichter, B. Kubis, M. Procura, and P. Stoffer, Phys. Lett. B 738, 6 (2014).

[22] G. Colangelo, M. Hoferichter, M. Procura, and P. Stoffer, J. High Energy Phys. 09 (2015) 074.

[23] G. Colangelo, M. Hoferichter, M. Procura, and P. Stoffer, Phys. Rev. Lett. 118, 232001 (2017).

[24] G. Colangelo, M. Hoferichter, M. Procura, and P. Stoffer, J. High Energy Phys. 04 (2017) 161.

[25] V. Pauk and M. Vanderhaeghen, Phys. Rev. D 90, 113012 (2014).

[26] V. Pascalutsa, V. Pauk, and M. Vanderhaeghen, Phys. Rev. D 85, 116001 (2012).

[27] J. Green, O. Gryniuk, G. von Hippel, H. B. Meyer, and V. Pascalutsa, Phys. Rev. Lett. 115, 222003 (2015).

[28] I. Danilkin and M. Vanderhaeghen, Phys. Rev. D 95, 014019 (2017).

[29] F. Hagelstein and V. Pascalutsa, Phys. Rev. Lett. 120, 072002 (2018).

[30] F. Hagelstein and V. Pascalutsa, arXiv:1907.06927.

[31] M. Hoferichter, B. Kubis, S. Leupold, F. Niecknig, and S. P. Schneider, Eur. Phys. J. C 74, 3180 (2014).

[32] P. Masjuan and P. Sanchez-Puertas, Phys. Rev. D 95, 054026 (2017).

[33] M. Hoferichter, B.-L. Hoid, B. Kubis, S. Leupold, and S. P. Schneider, Phys. Rev. Lett. 121, 112002 (2018).

[34] M. Hoferichter, B.-L. Hoid, B. Kubis, S. Leupold, and S. P. Schneider, J. High Energy Phys. 10 (2018) 141. 
[35] A. Gérardin, H. B. Meyer, and A. Nyffeler, Phys. Rev. D 100, 034520 (2019).

[36] G. Eichmann, C. S. Fischer, E. Weil, and R. Williams, Phys. Lett. B 797, 134855 (2019).

[37] R. García-Martín and B. Moussallam, Eur. Phys. J. C 70, 155 (2010).

[38] M. Hoferichter, D. R. Phillips, and C. Schat, Eur. Phys. J. C 71, 1743 (2011).

[39] B. Moussallam, Eur. Phys. J. C 73, 2539 (2013).

[40] I. Danilkin and M. Vanderhaeghen, Phys. Lett. B 789, 366 (2019).

[41] M. Hoferichter and P. Stoffer, J. High Energy Phys. 07 (2019) 073.

[42] I. Danilkin, O. Deineka, and M. Vanderhaeghen, arXiv: 1909.04158.

[43] J. Calmet, S. Narison, M. Perrottet, and E. de Rafael, Phys. Lett. 61B, 283 (1976).

[44] A. Kurz, T. Liu, P. Marquard, and M. Steinhauser, Phys. Lett. B 734, 144 (2014).

[45] G. Colangelo, M. Hoferichter, A. Nyffeler, M. Passera, and P. Stoffer, Phys. Lett. B 735, 90 (2014).

[46] J. Bijnens, N. Hermansson-Truedsson, and A. RodríguezSánchez, Phys. Lett. B 798, 134994 (2019).

[47] K. Melnikov and A. Vainshtein, Phys. Rev. D 70, 113006 (2004).

[48] A. Vainshtein, Phys. Lett. B 569, 187 (2003).

[49] M. Knecht, S. Peris, M. Perrottet, and E. de Rafael, J. High Energy Phys. 03 (2004) 035.

[50] M. Knecht, S. Peris, M. Perrottet, and E. de Rafael, J. High Energy Phys. 11 (2002) 003.

[51] A. Czarnecki, W. J. Marciano, and A. Vainshtein, Phys. Rev. D 67, 073006 (2003); 73, 119901(E) (2006).

[52] F. Jegerlehner and O. V. Tarasov, Phys. Lett. B 639, 299 (2006).

[53] J. Mondejar and K. Melnikov, Phys. Lett. B 718, 1364 (2013).

[54] W. A. Bardeen and W. K. Tung, Phys. Rev. 173, 1423 (1968); 4, 3229(E) (1971).

[55] R. Tarrach, Nuovo Cimento A 28, 409 (1975).

[56] V. A. Novikov, M. A. Shifman, A. I. Vainshtein, M. B. Voloshin, and V. I. Zakharov, Nucl. Phys. B237, 525 (1984).

[57] G. P. Lepage and S. J. Brodsky, Phys. Lett. 87B, 359 (1979).

[58] G. P. Lepage and S. J. Brodsky, Phys. Rev. D 22, 2157 (1980).

[59] S. J. Brodsky and G. P. Lepage, Phys. Rev. D 24, 1808 (1981).

[60] K. Melnikov and A. Vainshtein, arXiv:1911.05874.

[61] E. R. Arriola and W. Broniowski, Phys. Rev. D 74, 034008 (2006).

[62] E. R. Arriola and W. Broniowski, Phys. Rev. D 81, 094021 (2010).

[63] S. Peris, M. Perrottet, and E. de Rafael, J. High Energy Phys. 05 (1998) 011.

[64] M. Knecht, S. Peris, and E. de Rafael, Phys. Lett. B 443, 255 (1998).

[65] J. Bijnens, E. Gámiz, E. Lipartia, and J. Prades, J. High Energy Phys. 04 (2003) 055.
[66] G. Colangelo, F. Hagelstein, M. Hoferichter, L. Laub, and P. Stoffer, arXiv:1910.13432.

[67] P. Masjuan, E. Ruiz Arriola, and W. Broniowski, Phys. Rev. D 85, 094006 (2012).

[68] M. Tanabashi et al. (Particle Data Group), Phys. Rev. D 98, 030001 (2018).

[69] M. Knecht, A. Nyffeler, M. Perrottet, and E. de Rafael, Phys. Rev. Lett. 88, 071802 (2002).

[70] M. J. Ramsey-Musolf and M. B. Wise, Phys. Rev. Lett. 89, 041601 (2002).

[71] M. Acciarri et al. (L3 Collaboration), Phys. Lett. B 413, 147 (1997).

[72] P. Salvini et al. (OBELIX Collaboration), Eur. Phys. J. C 35, 21 (2004).

[73] R. Escribano, S. Gonzàlez-Solís, P. Masjuan, and P. Sanchez-Puertas, Phys. Rev. D 94, 054033 (2016).

[74] L. G. Landsberg, Phys. Rep. 128, 301 (1985).

[75] U.-G. Meißner, Phys. Rep. 161, 213 (1988).

[76] T. Feldmann, P. Kroll, and B. Stech, Phys. Rev. D 58, 114006 (1998).

[77] T. Feldmann, Int. J. Mod. Phys. A 15, 159 (2000).

[78] M. Acciarri et al. (L3 Collaboration), Phys. Lett. B 418, 399 (1998).

[79] H. J. Behrend et al. (CELLO Collaboration), Z. Phys. C 49, 401 (1991).

[80] J. Gronberg et al. (CLEO Collaboration), Phys. Rev. D 57, 33 (1998).

[81] P. del Amo Sanchez et al. (BABAR Collaboration), Phys. Rev. D 84, 052001 (2011).

[82] J. P. Lees et al. (BABAR Collaboration), Phys. Rev. D 98, 112002 (2018).

[83] M. Acciarri et al. (L3 Collaboration), Phys. Lett. B 501, 1 (2001).

[84] R. Ahohe et al. (CLEO Collaboration), Phys. Rev. D 71, 072001 (2005).

[85] M. Ablikim et al. (BESIII Collaboration), Phys. Rev. D 97, 072014 (2018).

[86] P. Achard et al. (L3 Collaboration), J. High Energy Phys. 03 (2007) 018.

[87] C. C. Zhang et al. (Belle Collaboration), Phys. Rev. D 86, 052002 (2012).

[88] E. Klempt and A. Zaitsev, Phys. Rep. 454, 1 (2007).

[89] P. Sanchez-Puertas (private communication).

[90] M. Knecht and A. Nyffeler, Phys. Rev. D 65, 073034 (2002).

[91] J. Prades, E. de Rafael, and A. Vainshtein, Adv. Ser. Dir. High Energy Phys. 20, 303 (2009).

[92] M. Davier, S. Descotes-Genon, A. Hocker, B. Malaescu, and Z. Zhang, Eur. Phys. J. C 56, 305 (2008).

[93] M. Beneke and M. Jamin, J. High Energy Phys. 09 (2008) 044.

[94] K. Maltman and T. Yavin, Phys. Rev. D 78, 094020 (2008).

[95] S. Narison, Phys. Lett. B 673, 30 (2009).

[96] I. Caprini and J. Fischer, Eur. Phys. J. C 64, 35 (2009).

[97] A. Pich, Prog. Part. Nucl. Phys. 75, 41 (2014).

[98] J. Leutgeb and A. Rebhan, arXiv:1912.01596.

[99] L. Cappiello, O. Catà, G. D’Ambrosio, D. Greynat, and A. Iyer, arXiv:1912.02779. 